primitiven Kulturen, im alten Ägypten und in gewissem Umfang auch im Mittelalter. In solchen Gesellschaften kann es wenige Reiche und viele Arme, wenige Mächtige und viele Unterdrückte geben, genau wie das (z. B. im Kapitalismus) bei Cesellschaften der Fall ist, in denen die Eigentumsrechte bei den Individuen als solchen liegen. ${ }^{197}$

Eingedenk der historischen Vorläufer kam Burnham angesichts seiner Erkenntnis, dass im "Regime der Manager Privatleute kein entsprechendes Eigentum besaßen und mit Blick auf die Frage, wie dann überhaupt ein Gruppe von Menschen eine herrschende Klasse bilden kann, zu folgender Antwort: »Nicht durch Eigentumsrechte, die sie als einzelne besitzen, werden die Manager die Kontrolle über die Produktionsmittel ausüben und bei der Verteilung bevorzugt werden, sondern mittelbar durch die Kontrolle über den Staat, der seinerseits die Produktionsmittel zu Eigentum besitzt und kontrolliert. $^{198}$

Der in der Studie The Managerial Revolution von Burnham entfaltete Kerngedanke der Konstituierung des oligarchischen Kollektivismus anstatt einer sozialistischen Gesellschaft stieß bei Koestler, Borkenau ${ }^{199}$ als auch - wie erwähnt - bei Orwell auf großes Interesse. Insbesondere hatte die burnhamsche Prognose, dass hinfort die Welt von drei großen Superstaaten beherrscht werden, die untereinander in einem permanenten Kriegszustand leben werden, vor allem für das totalitarismuskritische Buch Nineteen Eighty-Four Orwells eine außerordentliche Bedeutung. ${ }^{200}$

\title{
3.3 Zur Bedeutung der Renegaten für die Totalitarismustheorie
}

Mit The Totalitarian Enemy von Borkenau und The Managerial Revolution Burnhams lagen zu einem frühen Zeitpunkt totalitarismustheoretische Studien von zwei ehemaligen Kommunisten respektive Trotzkisten vor. Insbesondere die Studie von Borkenau avancierte zu einem Standardwerk der Totalitarismusforschung. Darüber hinaus

197 Burnham, Das Regime der Manager, S. $56 \mathrm{f}$.

198 Ebd., S. 92.

199 Siehe Franz Borkenau, Die Revolution der Manager, in: Merkur 3 (1949), H. 5, S. 487-494.

200 Ceorge Orwell schrieb in dem Aufsatz James Burnham and the Managerial Revolution aus dem Frühjahr 1946 zustimmend: »Die neuen >Manager-Gesellschaften werden nicht aus einem Flickenteppich kleiner, unabhängiger Staaten, sondern aus großen Superstaaten bestehen, die sich um die industriellen Hauptzentren in Europa, Asien und Amerika konzentrieren. Diese Superstaaten werden untereinander um den Besitz der noch nicht mit Beschlag belegten Teile der Welt kämpfen, aber sie werden wahrscheinlich nicht in der Lage sein, einander völlig zu besiegen. Im Innern wird jede Gesellschaft hierarchisch sein mit einer Begabtenaristokratie an der Spitze und einer Masse von Halbsklaven an der Basis.« (Zit. n. Hans-Christoph Schröder, George Orwell. Eine intellektuelle Biographie, München 1988, S. 193) - Kritisch äußerte sich Orwell hinsichtlich Burnhams Vorstellung, dass dieser die Stabilität der totalitären Herrschaftsregime in der Sowjetunion und in Deutschland überschätzt und die zweifelsohne vorhandenen systemimmanenten Zerstörungskräfte in ebendiesen Staaten mehr oder weniger unterschätzt. Mit Blick auf die Sowjetunion prognostizierte Orwell, dass sich das Regime unter Stalin entweder demokratisieren werde oder ganz verschwindet. Denn, so Orwell: »Das riesige, unbesiegbare, ewigwährende Sklavenreich, von dem Burnham zu träumen scheint, wird nicht errichtet werden, oder, wenn es errichtet wird, wird es nicht dauern, weil die Sklaverei nicht länger mehr eine stabile Grundlage für die menschliche Gesellschaft darstellt.« (Zit. n. ebd., S. 195) 
enthielten die zu einem frühen Zeitpunkt, d.h. die im Kontext des Spanischen Bürgerkrieges bis Anfang der 1950er-Jahre, entstandenen autobiografischen Renegatenberichte neben ersten Informationen über die Repressionen und über den »Großen Terror « in der Sowjetunion erste totalitarismustheoretische Ansätze, weil in zahlreichen Büchern explizit oder implizit ein Vergleich zwischen dem Nationalsozialismus und dem Stalinismus gezogen wurde, ${ }^{201}$ sodass hier eine »Embryonalform der Totalitarismustheorie« (Kraushaar) vorlag.

So zog beispielsweise der ehemalige jüdisch-österreichische Kommunist Alexander Weißberg-Cybulski, der im Kontext der »Großen Säuberung« im März 1937 in der Sowjetunion verhaftet und dann im Zuge des Hitler-Stalin-Paktes im Frühjahr 1940 vom russischen Geheimdienstapparat an die deutsche Gestapo ausgeliefert wurde, in seinem autobiografischen Erfahrungsbericht Im Verhör. Ein Überlebender der stalinistischen Säuberungen berichtet explizit den Vergleich: »Wir fuhren durch das verwüstete Polen auf Brest-Litowsk zu. An der Bugbrücke erwartete uns der Apparat des anderen totalitären Systems in Europa, die deutsche Gestapo. ${ }^{202}$ Zudem rückten bei Weißberg-Cybulski die für die totalitären Herrschaftssysteme des Nationalsozialismus und des Sowjetkommunismus eine zentrale Rolle einnehmenden Organe der staatlichen Geheimpolizei in den Mittelpunkt des Vergleichs. In Bezug auf die Folter bei den persönlich erfahrenen Verhören stellte er hierzu fest:

Die GPU erreichte ihr Ziel zumeist durch die Angst vor der drohenden Mißhandlung, in selteneren Fällen durch wirkliche Foltern. Sie waren sehr sparsam mit den Schlägen. (Darin unterschieden sie sich von den Cestapoleuten, wie mich sechs Jahre später mein Aufenthalt im Pawiak, dem Zentralgefängnis der Warschauer Cestapo, lehrte. Die GPU war viel klüger als die deutsche Geheimpolizei). ${ }^{203}$

Der ehemalige Kommunist Walter G. Krivitsky wiederum verglich die »Parteisäuberung « Stalins ausdrücklich mit der »blutigen Säuberung vom 30. Juni 1934«, der sogenannten Röhmaffäre, durch Hitler. ${ }^{204}$ Bei Buber-Neumann tauchte der Vergleich expressis verbis schon im Buchtitel auf. Die 1949 veröffentlichten Erinnerungen Als Gefangene bei Stalin und Hitler thematisierten hierbei wie gesehen keineswegs nur ausschließlich totalitäre Erfahrungen. ${ }^{205}$ Im Mittelpunkt des Buches stand primär die Institution, in der das Ziel des totalitären Staates, die totale Herrschaft über das einzelne Individuum, erfüllt schien, wo alles erlaubt war und beispielsweise für Arendt "schlechthin alles möglich« war und das Wesen des Totalitarismus verkörperte: die Konzentrationslager. ${ }^{206}$

201 Siehe hierzu Rohrwasser, Totalitarismustheorie und Renegatenliteratur, S. 105-116.

202 Alexander Weißberg-Cybulski, Im Verhör. Ein Überlebender der stalinistischen Säuberungen berichtet, Wien/Zürich 1993, S. 335; die deutsche Originalausgabe erschien 1951 unter dem Titel Hexensabbat. Die Cedankenpolizei-Die große Tschistka im Verlag der Frankfurter Hefte. Unter dem Titel Das Grosse Verhör. Aus der Praxis des NKWD erschien ein Auszug des Buches in Der Monat (3 [1951], H. 35, S. 490-502).

203 Ebd., S. 227.

204 Krivitsky, Ich war in Stalins Dienst!, S. 203 f.

205 Siehe hierzu Kap. II.2.1.

206 Siehe Arendt, Elemente und Ursprünge totaler Herrschaft, S. 676-702. 
Die zentrale Bedeutung der Renegaten für die Totalitarismustheorie war neben dem frühzeitig gezogenen expliziten oder impliziten Vergleich zwischen dem $\mathrm{Na}$ tionalsozialismus und dem Stalinismus, die in zahlreichen autobiografischen Erfahrungsberichten der ehemaligen Kommunisten enthalten waren, ${ }^{207}$ indes einem wissenschaftlichen Umstand geschuldet. Ohne die Renegatenliteratur und ohne die Veröffentlichungen der früheren Kommunisten über das sowjetische Herrschaftsregime wären die »klassischen« Studien Elemente und Ursprünge totaler Herrschaft von Arendt, Totalitäre Diktatur von Friedrich oder auch The Permanent Purge von Brzezinski, schwer vorstellbar. ${ }^{208}$ Die ehemaligen Anhänger des Kommunismus wie Arthur Koestler, Boris Souvarine, Margarete Buber-Neumann, Franz Borkenau, Ruth Fischer, Manès Sperber, Jan Valtin, Wiktor Krawtschenko, Anton Ciliga, David J. Dallin, Boris Nikolajewski, Alexander Weißberg-Cybulski oder Walter G. Krivitsky besaßen die >Insiderkenntnisse destiniert, bis zum Zeitpunkt der Veröffentlichung der »klassischen« Totalitarismusstudien den sowjetischen Totalitarismus zu analysieren. Diese kleine Gruppe von ehemaligen kommunistischen Intellektuellen, in vielen Fällen führend im Parteiapparat aktiv, kannte die Natur des Kommunismus aus eigener Erfahrung und war deshalb besonders geeignet, den Herrschaftscharakter des sowjetischen Staates zu beschreiben und nicht zuletzt den »Großen Terror « unter Stalin zu thematisieren und zu analysieren.

Beispielsweise enthielt Koestlers Roman Sonnenfinsternis eine erste Erklärung für die in den Nachkriegsjahren gemeinhin nicht verstandenen »Geständnisse« der Angeklagten, d. h. führenden Mitgliedern der Kommunistischen Partei, in den drei "großen« öffentlichen Moskauer Schauprozessen, für Verbrechen, die sich nicht begangen hatten. Krawtschenko gab in Ich wählte die Freiheit detailliert Auskunft über die von Stalin im Zuge der Moskauer Prozesse in Gang gesetzte »Massensäuberungsaktion« in der Sowjetunion, von der Millionen Menschen in der gesamten Gesellschaft und den Partei- und Staatsapparaten betroffen waren, die entweder in die Konzentrationslager verschleppt oder direkt erschossen wurden. Und Weißberg-Cybulskis Buch Hexensabbath enthielt neben der Beschreibung und der Analyse eines »Tätertypus« und der Terrorinstitution des NKWD zudem die Beschreibung eines zentralen Motives für die »Verfolgungslogik« des sowjetischen Geheimdienstes, dass später von Arendt als »Schneeballsystem« angesehen und mit der analytischen Kategorie »guilt by associa-

207 Koestler kam in seinem post festum geschriebenen Nachwort zur Sonnenfinsternis im Zusammenhang mit der Tötung der zentralen Figur des Romans zu der gewissermaßen totalitarismustheoretischen Erkenntnis: »Als seine eigenen Leute ihn verhaften kommen, schläft Rubaschow noch und träumt von seiner letzten Verhaftung im Land des Feindes; in der Schlaftrunkenheit ist er sich nicht klar, welcher der beiden feindlichen Diktatoren es diesmal auf ihn abgesehen hat und welcher der beiden allgegenwärtigen Öldrucke über seinem Bett hängt. Merkwürdigerweise schrieb ich diese symbolische Cleichsetzung der beiden totalitären Regime (die als Leitmotiv in den letzten Zeilen des Buches wieder auftaucht) ein Jahr vor dem Hitler-Stalin-Pakt, als ich in meinem bewußten Cedanken noch mit den Sowjets sympathisierte und jede Andeutung einer Ähnlichkeit zwischen Sowjetrußland und Nazideutschland entrüstet zurückgewiesen hätte.« (Sonnenfinsternis, S. 227; Hervorh. des Verf.)

208 Zur Bedeutung der Renegatenliteratur für die Analyse des stalinschen Totalitarismus in der Studie von Hannah Arendt siehe Rohrwasser, Totalitarismustheorie und Renegatenliteratur, S. 115. 
tion $\aleph^{209}$ als ein Schlüsselprinzip für den stalinistischen Terror erklärt wurde. Die Renegaten lieferten den »empirischen Nachweis« über den Terror in der Sowjetunion. Anders gesagt: Die wirkliche Geschichte des sowjetischen Kommunismus wurde von Exkommunisten geschrieben. ${ }^{210}$

Die Renegaten und speziell die ehemaligen kommunistischen Intellektuellen unter ihnen untersuchten frühzeitig in ihren Büchern die Genesis der totalitären Herrschaft in der Sowjetunion unter Stalin. Sie beleuchteten das zentrale Problem der politischen Linken, warum die mit »emanzipatorischem Impetus « angetretene kommunistische Bewegung unter der Führung Lenins nicht die »Befreiung des Menschen« und die »Abschaffung von menschlicher Ausbeutung« erreichte, sondern zu einer Einparteienherrschaft und zu Ergebnissen führte, die prima vista mit dem Ziel der revolutionären Bewegung nichts zu tun hatte. Bei den Renegaten wie Borkenau, Orwell, Burnham oder auch Koestler - die, wie erwähnt, die jeweiligen Buchveröffentlichungen rezipierten und/oder rezensierten sowie sich gegenseitig beeinflussten - reifte die politische und theoretische Erkenntnis, dass die Abschaffung der kapitalistischen Gesellschaftsformation nicht zum »sozialistischen Paradies« führte und nicht das Ende der »Herrschaft des Menschen über den Menschen« bedeutete. ${ }^{211}$

Die Renegaten konnten sozusagen der Totalitarismustheorie den empirischen Nachweis liefern, dass es sich beim kommunistischen Sowjetstaat unter Stalin um den ersten totalitären Einparteienstaat im 20. Jahrhundert handelte. Das »Stalin-Regime« besaß der Erkenntnis der Renegaten zufolge eine besondere, d.h. neuartige Herrschaftsform und unterschied sich von früheren staatlichen Unterdrückungsformen qualitativ und war etwas noch nie Dagewesenes. Die ehemaligen Kommunisten legten in ihren autobiografischen Erfahrungsberichten und den (theoretisch-)historischen Untersuchungen zum Sowjetkommunismus den beschreibenden bzw. analytischen Fokus explizit oder implizit auf die Denkfigur der "permanenten Revolution« (Neumann). Im Gegensatz zu den bis dahin bekannten, eher konservativen, statischen Herrschafts- bzw. Unterdrückungsformen wie der Despotie, der Tyrannis oder dem Autoritarismus handelte es sich nämlich beim Totalitarismus stalinistischer Provenienz um ein »Bewegungsregime«. Um das ideologische Endziel des politisch-sozialen Projektes der »klassenlosen Gesellschaft« in der Sowjetunion - und á la longue der kommunistischen Weltherrschaft - zu realisieren, setzte Stalin Ende der 1920er-1 Anfang der 1930er-Jahre, vor dem Hintergrund des Zieles, die Industrialisierung der Sowjetunion zu beschleunigen, mit dem ersten »Fünfjahresplan« eine "permanente Revolution von oben « und eine »Terrorwelle« in Gang. Die Renegaten thematisierten aus eigener Erfahrung, wie auf der Grundlage des "geschlossenen Denksystems« (Koestler) des Kommunismus Millionen von Bauern unter dem stalinschen Ideologem "Liquidierung der Kulaken als Klasse« enteignet, ums Leben kamen und in die Konzentrations- bzw. Arbeitslager verschleppt wurden. Darüber hinaus wurden auch Mitte der 1930er-Jahre im »Großen Terror« Millionen sowjetischer Bürger, die nicht Mitglied der Kommunistischen Partei waren, von der »revolutionären Diktatur« entweder umgebracht oder in die sowjetischen Lager verschleppt, was mit traditioneller Staatsräson und sogenannter normaler staatlicher Machtpolitik nicht mehr zu er-

209 Arendt, Elemente und Ursprünge totaler Herrschaft, S. 523.

210 Furet, Das Ende einer Illusion, S. 191.

211 Franz Borkenau, Die Revolution der Manager, in: Merkur 3 (1949), H. 5, S. 489. 
klären war. Im Zuge der "Revolution von oben« ließ Stalin im Kontext der Moskauer Schauprozesse nicht nur »objektive Gegner« bzw. »subjektive Gegner« als »gegenrevolutionäre Verräter« öffentlich verurteilen, sondern zudem eine groß angelegte Parteisäuberung durchführen. Von der »Massensäuberung« nach dem »Kirow-Mord « ${ }^{212} \mathrm{im}$ Dezember 1934 waren nicht nur führende Parteifunktionäre der KPdSU in der Bürokratie und der Armee betroffen. Das, was später Arendt als Kennzeichen der »neuen Staatsform « $^{213}$ und als Wesen des totalitären Herrschaftssystems ansah, dass nämlich der ideologisch motivierte in Gang gebrachte Terror immer neue Bevölkerungsgruppen erfasste, thematisierten zum Beispiel Buber-Neumann oder Weißberg-Cybulski ausdrücklich in ihren Büchern. Die innerparteiliche Terrorwelle wiederum erfasste in der Sowjetunion nicht nur Mitglieder der KPdSU, sondern unter anderem auch überzeugte Kommunisten und Politemigranten aus Deutschland, Österreich und Polen, die unter der Anklage »konterrevolutionäre, terroristische, trotzkistische Elemente« Opfer der stalinistischen »Verfolgungslogik« wurden, obwohl keine Wirtschaftskrise und mithin keine »objektiven « Gründe vorlagen und sie keine Opposition darstellten. ${ }^{214}$ Die Renegaten nahmen vorweg, was später beispielsweise das Schlüsselwerk von Arendt zum Ausdruck brachte: dass es sich beim (sowjetischen) Totalitarismus um eine »Schreckensherrschaft « einer Parteielite handelte, dessen Wesen sich in erster Linie im Faktum des Terrors eines Geheimdienstapparates und der Konzentrationslager enthüllte.

Zusammenfassend kann festgestellt werden, dass es nach dem "Schock « des deutsch-sowjetischen Nichtangriffspaktes auch und vor allem Renegaten waren - wie zum Beispiel in der Zeitschrift Die Zukunft-, die öffentlich den Vergleich zwischen Hitler und Stalin zogen. Zudem stammte vom Exkommunisten Borkenau eine der ersten komparativ angelegte Studien zum Nationalsozialismus und zum Sowjetkommunismus, die die totalitäre Herrschaftsform hervorhob und die in der Geschichte der Totalitarismustheorie einen zentralen Platz einnimmt. Borkenaus 1940 veröffentlichte Studie The Totalitarian Enemy enthielt analytische Erkenntnisse, die hinfort für die »klassischen« Totalitarismustheorien von essenzieller Bedeutung waren. Denn für Borkenau war der Totalitarismus eine spezifische Herrschaftsform des 20. Jahrhunderts und entstand aus der durch den Ersten Weltkrieg ausgelösten politischen, sozialökonomischen und ideologischen Krise und ging mithin aus einer nichttotalitären Massengesellschaft hervor. Insbesondere mit Blick auf die Genesis des Nationalsozialismus insistierte der marxistische Theoretiker und ehemalige Kommunist auf die Tatsache, dass die »Machtergreifung« der hitlerschen Massenbewegung nur möglich

212 Siehe Krivitsky, Ich war in Stalins Dienst!, S. 203, sowie Kravchenko, Ich wählte die Freiheit, S. 222.

213 Vgl. auch Paul Sering (d. i. Richard Löwenthal), Jenseits des Kapitalismus. Ein Beitrag zur sozialistischen Neuordnung, Lauf bei Nürnberg 1946, S. $117 \mathrm{f}$.

214 In Hexensabbat fragte sich Alexander Weißberg-Cybulski (Ein Überlebender der stalinistischen Säuberungen berichtet): »[W]arum sie plötzlich aus heiterem Himmel Hunderttausende verhafteten. Es war doch nichts im Lande geschehen. Keinerlei Krise im Dorf. Keine verstärkte Kriegsgefahr. Alles ging verhältnismäßig gut. Jedenfalls viel besser als vier Jahre vorher, und doch hatten die Verhaftungen im großen Stil erst im Jahre 1936 begonnen.« (Zit. n. Weißberg-Cybulski, S. 125) - Später stellte Arendt (Elemente und Ursprünge totaler Herrschaft) mit Blick auf den »furchtbare[n] Terror« zu Beginn der 1930er-Jahre das gleiche Phänomen fest: »Nicht in den zwanziger Jahren und zu Zeiten des Bürgerkrieges in Rußland, sondern nach 1930, als alle gegenrevolutionäre wie innerparteiliche Opposition restlos liquidiert war, brach die wirkliche Schreckensherrschaft in Rußland an.« (S. 616) 
war, weil die alte politische Klasse sich außerstande zeigte, die genuinen Probleme der Weimarer Republik zu lösen. Anders gesagt: Auch in Deutschland ist der Nationalsozialismus "nicht vom Himmel gefallen«, wie bereits vor ihm Silone in seiner Untersuchung über die historische Genese des italienischen Faschismus herausfand, sondern aufgrund der komplexen politischen und gesellschaftlichen Krise der Weimarer Republik.

Für Borkenau war der Totalitarismus bereits qua definitionem bzw. Selbstverständnis der natürliche Gegner, genauer: der existenzielle Feind der liberalen oder pluralistischen Demokratie, mit dem eine friedliche Koexistenz ausgeschlossen war. Einerseits weil die Feindsuche zur Obsession und zum circulus vitiosus des Totalitarismus wurde und mithin ein friedliches Zusammenleben mit anderen, zumal demokratischen Staaten nicht möglich war. Andererseits war der Totalitarismus dem ideologischen Selbstverständnis nach die Antithese zu den Demokratien, da er dem Staat, der Partei und der totalitären Massenbewegung den absoluten Vorrang einräumte und er sich das "Ende des Subjektes « und die radikale Beseitigung aller persönlichen sowie staatsfreien Freiheitsrechte zum Ziel gesetzt hatte.

Neben der konkreten Beschreibung und Analyse des stalinistischen Herrschaftsregimes lag die Bedeutung der Renegaten für die »klassischen« Totalitarismustheorien zudem vor allem darin, dass sie den Fokus einerseits auf die für die einzelnen Studien zentralen Kategorien wie »Einparteienregime«, »Geheimpolizei«, »totalitäre Ideologie«, »(permanenter) Terror«, »Konzentrationslager«, »Feindobsession«, »permanente Revolution« etc. richteten. Andererseits haben sie die differentia specifica zwischen dem italienischen Faschismus und dem Nationalsozialismus sowie dem Sowjetkommunismus hervorgehoben. Schon Borkenau subsumierte in The Totalitarian Enemy ausdrücklich nur den Nationalsozialismus und den Sowjetkommunismus unter das Totalitarismusmodell. Zudem arbeiteten die Renegaten in den autobiografischen Erfahrungsberichten ex negativo die qualitativen Unterschiede heraus, die zwischen einem totalitären und einem faschistischen Herrschaftsregime bestanden - bei allen zweifelsohne vorhandenen Gemeinsamkeiten -, dass nämlich der Totalitarismus eine Verbrechensdimension entwickelte, die dem italienischen Faschismus unter Mussolini gewissermaßen fremd war. Insbesondere der amerikanische Ex-Trotzkist Macdonald unterstrich bereits 1944 explizit und implizit den einzigartigen Herrschaftscharakter des nationalsozialistischen Totalitarismus in Bezug auf die Ermordung der europäischen Juden. Vergleichbare Verbrechen hatte der italienische Faschismus nicht begangen. Vor diesem Hintergrund gingen Arendt und Friedrich später in ihren »klassischen« totalitarismustheoretischen Studien von den qualitativen Differenzen zwischen dem italienischen Faschismus auf der einen Seite und dem nationalsozialistischen sowie sowjetkommunistischen Totalitarismus aus.

\subsection{Die Rolle der Renegaten im Monat}

Vor dem Hintergrund ihrer frühzeitigen theoretischen und politischen Auseinandersetzung mit den totalitären Herrschaftssystemen des Nationalsozialismus und des Sowjetkommunismus kann auch im Folgenden die spezielle Rolle der zahlreichen ehemaligen kommunistischen (Partei-)Intellektuellen in der Zeitschrift Der Monat verdeutlicht werden. Aufgrund der zum Teil frühzeitig einsetzenden Ausein- 